\title{
Abdi-Herrle, Sasan: Mediale Themensetzung in Zeiten von Web 2.0. Wer beeinflusst wen? Das Agenda-Setting-Verhältnis zwischen Twitter und Online-Leitmedien
}

\author{
Baden-Baden: Nomos Verlagsgesellschaft 2018. 198 Seiten. Preis: \\ $€ 20,90$
}

\author{
Hanan Badr \\ Online publiziert: 11. März 2020 \\ (C) Der/die Autor(en) 2020
}

Die zunehmende Nutzung von Twitter und anderen sozialen Medien seitens Politiker, Prominenter und auch Journalisten scheint eine Erosion der Glaubwürdigkeit und der Autorität der traditionellen Massenmedien zu bestätigen. Doch gleichzeitig können die sozialen Medien ohne die klassischen ,alten“ Medien nicht überleben: die Debatten, die da ausgelöst werden, erhalten einen Resonanzraum in und das Input-Material, das da kommentiert und geteilt wird, stammt zu beachtlichen Teilen aus klassischen journalistischen Medien. Genau diese Prozesse der gegenseitigen Abhängigkeit und Beeinflussung schaut sich die Dissertationsschrift von Sasan Abdi-Herrle genauer an.

Abdi-Herrle erinnert in den ersten Seiten seines Buches daran, dass Donald Trump nicht der erste Politiker war, der die klassischen Massenmedien umgangen hat. Lange vor ihm hat der frühere US-Präsident Barack Obama beispielsweise die Ergebnisse der Präsidentschaftswahlen seiner zweiten Amtszeit im November 2012 auf Twitter bekanntgegeben. Privatpersonen und Journalisten konnten damit gleichzeitig auf demselben Wissensstand sein. Das kündigte die neue Ära und einen Paradigmenwechsels in der politischen Kommunikation an.

In dem Buch geht es zunächst um die Frage, wie sich Twitter und die Online-Auftritte klassischer Leitmedien wechselseitig in der heutigen komplexen und vernetzten politischen Kommunikation beeinflussen. Gerade im deutschsprachigen Raum fehle Literatur zu diesem Thema, anders als im US-amerikanischen Raum. Darüber hinaus fragt die Studie in der zweiten Forschungsfrage danach, unter welchen Bedingungen Twitter die Agenda von Online-Leitmedien beeinflusse.

Prof. Dr. H. Badr ( $\triangle)$

Institut für Publizistik- und Kommunikationswissenschaft, Freie Universität Berlin,

Garystr. 55, 14195 Berlin, Deutschland

E-Mail: hanan.badr@fu-berlin.de 
Theoretisch verortet der Autor die Arbeit in den Agenda-Setting-Theorien in einem hybriden Mediensystem. Dabei verknüpft das Buch die klassischen Arbeiten in der Agenda-Building Forschung, z. B. von Cobb, Elder und Pfetsch, mit dem neueren theoretischen Zugang zu ,hybriden Mediensystemen“, der von Andrew Chadwick 2013 vorgelegt wurde. Die Studie positioniert die neuen Medien als Herausforderer, deren Rolle im Zuge der Hybridisierung des Mediensystems zugenommen habe, bei gleichzeitigem Verlust von Deutungshoheit und Nutzungszahlen bei klassischen Medien.

Das empirische Herzstück der Agenda-Forschung ist der Vergleich von zwei Agenden im Medien-Politik-Publikum Dreieck. Ein spezieller, klassischer Strang stellt jedoch das Intermedia-Agenda-Setting dar, wo nur Medienagenden untersucht und verglichen werden. Die Grundannahme der Intermedia-Agenda-Setting Forschung besagt, dass ,bestimmte Leitmedien mit ihrer Themensetzung die Agenda von anderen, weniger relevanten Medien bestimmen“" (S. 33). Die vorliegende Studie bedient sich dieser Annahme, um das Agenda-Setting-Verhältnis zwischen OnlineMedien und Twitter im deutschsprachigen Raum zu rekonstruieren und mittels Korrelation der Themenfrequenzen zu untersuchen. Das elitär-angelegte Twitter und die Online-Ausgaben der Qualitätspresse sind dabei bewusst als Untersuchungsmaterial ausgewählt.

Die empirische Basis der Untersuchung bilden zwei Fallbeispiele: TTIP und die Ukraine-Krise. Beide Themen sind während der Europawahl 2014 aufgekommen. Über die statistischen Korrelationen zwischen Online-Medienagenda und TwitterAgenda hinaus berücksichtigt die Studie Nachrichtenfaktoren sowie die Rolle der Nutzerstrukturen in Twitter-Netzwerken als Erklärungselemente.

Die Ergebnisse tragen wesentlich zum Verständnis der Intermedia-Agenda-Setting Prozesse im deutschsprachigen Raum bei, denn sie zeigen, dass journalistische Online-Medien immer noch ihre Leitmedienfunktion gegenüber Twitter behalten haben. Den Befunden zufolge finde ein Trickle-Down-Effekt statt, bei dem die Artikel der Online-Medien in die Twittersphäre einsickerten und somit eine diskursive Wirkung ausübten. Anders als der Diskurs in den Online-Leitmedien sind die Debatten auf Twitter dagegen zugespitzter, gerade bei polarisierenden Themen wie TTIP. Ähnlich wie im US-amerikanischen Raum sei eine Zeitverzögerung zwischen OnlineMedien und Twitter-Posts von $24 \mathrm{~h}$ auch im deutschsprachigen Raum zutreffend.

Die empirischen Ergebnisse zeigen, dass selbst in Zeiten von Web 2.0 klassische theoretische Zugänge wie Nachrichtenwerttheorie relevant sind. Die Analyse der Nachrichtenfaktoren ist einigermaßen überraschend: anstelle der erwarteten Faktoren Prominenz und Personalisierung als Ausgangspunkt für ein Intermedia-AgendaSetting dominieren Faktoren wie Kontroverse, Reichweite und Überraschung. In Anlehnung an Bourdieus Konzept vom sozialen Kapital kommt die Studie zum Ergebnis, dass je mehr Netzwerk-Autoritäten ein Twitter-Thema behandeln, d.h. zentraler und bekannter die Nutzer in ihren Netzwerken sind, desto mehr greifen es die Online-Medien auf. Es bleibt aber in dem Forschungsdesign offen, inwiefern die Online-Medien mit ihren offline Leitmedien interagieren, und inwiefern dabei eine offline-online Interaktion stattfindet. Auch wenn die empirische Analyse von 2014 stammt und ein paar Jahre zurück liegt, liefert sie wichtige Indizien für die Wechselwirkungen zwischen Twitter und Online-Leitmedien im deutschsprachigen 
Raum. Im Fazit reflektiert der Autor selbst offen über die Schwächen dieser Arbeit, die z.B. keine kausalen Zusammenhänge herstellen kann oder von einer größeren Anzahl an Fallstudien in der Aussagekraft profitieren würde.

Aus theoretischer Perspektive belegt das Buch, dass die bisherigen Instrumente und Theoreme der politischen Kommunikationsforschung sich weiterhin für die Analyse von hybriden Mediensystemen eignen, auch wenn sich Agenda-SettingProzesse vielfältiger gestalten.

Funding Open Access funding provided by Projekt DEAL.

Open Access Dieser Artikel wird unter der Creative Commons Namensnennung 4.0 International Lizenz veröffentlicht, welche die Nutzung, Vervielfältigung, Bearbeitung, Verbreitung und Wiedergabe in jeglichem Medium und Format erlaubt, sofern Sie den/die ursprünglichen Autor(en) und die Quelle ordnungsgemäß nennen, einen Link zur Creative Commons Lizenz beifügen und angeben, ob Änderungen vorgenommen wurden.

Die in diesem Artikel enthaltenen Bilder und sonstiges Drittmaterial unterliegen ebenfalls der genannten Creative Commons Lizenz, sofern sich aus der Abbildungslegende nichts anderes ergibt. Sofern das betreffende Material nicht unter der genannten Creative Commons Lizenz steht und die betreffende Handlung nicht nach gesetzlichen Vorschriften erlaubt ist, ist für die oben aufgeführten Weiterverwendungen des Materials die Einwilligung des jeweiligen Rechteinhabers einzuholen.

Weitere Details zur Lizenz entnehmen Sie bitte der Lizenzinformation auf http://creativecommons.org/ licenses/by/4.0/deed.de.

Dr. Hanan Badr ist Vertretungsprofessorin in am Institut für Publizistik- und Kommunikationswissenschaft der Freien Universität Berlin. 